\title{
声波感应论
}

\section{Acoustic Induction}

张海

\author{
Hai Zhang
}

【摘要】声音的形成必须具备 3 个条件: 声源、中间介质和感应器官。没有声源振动, 一 切声音都不可能产生, 所以声源是声音形成的前提条件; 没有中间介质传递声波能量, 声 音频率就不可能到达感应器官, 所以中间介质是连接声源和感应器官的纽带; 没有感应器 官就不能产生声音效果, 声波信息如果不能被接收, 声源和中间介质的一切工作就成了徒 劳。可见, 声音产生的这 3 个环节缺一不可。

【Abstract】The formation of sound must have three conditions: sound source, intermediate

\section{XXXXXXXX181X}

中国·河北 张家口 075636

\section{XXXXXXXX181X,}

Zhangjiakou, Hebei, 075636, China medium and sensory organs. Without sound source vibration, all sounds are impossible to produce, so the sound source sounds the preconditions; without the intermediate medium transmitting the sound wave energy, the sound frequency cannot reach the sensing organ, so the intermediate medium is the link connecting the sound source and the sensing organ; Without the sensory organ, no sound effect can be produced. If the sound wave information cannot be received, all the work of the sound source and the intermediate medium becomes futile. It can be seen that these three links of sound are indispensable.

【关键词】固定声源; 移动声源; 声源性质; 声源形式; 介质形态; 声波振动; 听觉神经

【Keywords】fixed sound source; moving sound source; sound source properties; sound source form; medium form; acoustic vibration; auditory nerve

【DOI 】10.36012/sde.v1i2.199

\section{1 引言}

宇宙中充满了各式各样的物质, 不但种类繁多,而且数量 庞大, 除形体物质外, 还有量子物质充斥期间。每个物质之间 通过摩擦、碰撞都可能产生声音。不单是木、石类物质的摩擦, 就连水分子、空气分子的摩擦都会形成声音。所以,任何物质 都是声源物质, 宇宙声音就会随时随地、不间断地发生,之所 以人们会感到片刻安宁, 那是因为有些声源离得较远, 再加上 声音在空气中的传播速度只有 $300 \mathrm{~m} / \mathrm{s}$, 还没等到达身边就散 失了; 再者, 人们能感知的声波频率为 $20 \sim 2000 \mathrm{~Hz}$, 超出这个 频率范围的声音, 只能被其他生物感知, 人类却无能为力。

\section{2 声源}

声源指的是发声物质间的相互摩擦，它的性质和作用方 式, 对于发声效果具有重要影响, 也就是体现在音质音色和声 波频率大小上的区别[1]。
声源性质就是声源的物质性, 一般指 2 个或多个摩擦物 质的属性, 如金属物质、岩石物质、草本物质、流体物质、量子 物质等。每种属性的物质产生声音的音质都有区别: 有的清 脆、有的混浊; 有的高六、有的低沉。所以, 声源的物质性就决 定了声音的基本性质。每种属性的物质都有特定的声音性质, 人们从中可以辨识发声物质的基本属性。所有的物质都可能 成为声源物质, 所有的声源物质都有着不同的音质效果和频 率信息。

声源形式是指声源物质的质量、形状和状态。有的物质质 量较大, 有的物质形状不规则, 而多数物质是处于相对运动状 态, 以此, 人们就可以感受声波能量强弱不同和频率节奏快慢 的声音信息。根据声源的物质状态, 可以将声源分为固定声源 和移动声源 2 大类。固定声源是指发声物质的位置固定不动, 与接声物质保持一定的距离, 或接声物质向声源物质靠近和 远离, 以发声物质为中心; 移动声源是指发声物质的位置发生 
理论实践 Theoretical Practice

变化, 或向接声物质靠近或向接声物质远离, 出现距离的加大 或缩小, 以接声物质为中心。

可以肯定的是, 所有的声源物质之间, 通过相互摩擦, 都 可以产生大小不等的能量和频率不同的声音信息。所以, 声 音信息的传递也就等于是能量的传递, 其能量以振波形式传 递, 从声源发出时最强, 依次减弱。这样, 物质的属性和摩擦 方式就起了关键作用。一般情况下, 质量较大的金属物质, 产 生声波的能量就强, 传播距离也远; 质量很小的量子物质, 产 生声波的能量很弱, 传播距离也近, 这就很难被生物感知。如 果质量较小的单个物质之间相互摩擦或碰撞, 其生成的声波 能量就很微弱, 易于形成次声波; 如果质量较大的多数物质 之间发生摩擦, 其生成的声波能量就非常强大, 以利于超声 波的形成。

\section{3 中间介质}

中间介质是连接声源和感应器官的纽带, 是传递声波能 量的介质物质。

自然界中的任何物质都可以暂时充当中间介质，它们的 性质和形状存在很大差异, 所产生的声波能量程度强弱不一。 根据需要的不同, 人们把中间介质分为自然介质、人工介质和 选择介质 3 大类。自然介质就是在自然界中客观存在、不被人 类加工和生物选择的介质物质,如岩石、水、空气等; 人工介质 是指经过人类加工制作可以充当的介质物品, 如听诊器、助听 器、电线、纸筒等; 选择介质就是根据听者的需要, 有必要进行 选择的介质物质, 如选择铁轨、选择铁丝、选择管道等。介质物 质的能量, 对于声波能量的传输, 具有方向性改变的作用, 其 能量的大小会随着介质物质的体积和质量而缩小，介质能量 越大, 阻止的声波能量越多, 听到的声音就越小。所以, 声音在 空气中传播的就远,在水中或树木中传播的就近。介质物质的 表面平整度, 也会影响声波的传递速度: 表面光滑平整的介质 物质, 传递声波的速度就快, 易于出现回声现象; 表面粗敉凹 凸的介质物质, 传递声波的速度就慢, 吸收声波的能力就强, 所以隔音板应选择表面粗䊁的物质。因此, 可以得出结论: 声 波速度以直线传播最快, 以曲线传播较慢。

中间介质物质对于声音的传播发挥着重要作用, 它衔接 了声源和感应器官, 充当了它们的媒介, 既可以让声波能量变 大也可以变小, 既可以让声音迅速传递也可以延缓进程。在真 空条件下, 声波信息就无法传递, 声音效果也不能实现, 因为 失去了空气分子的中间介质作用, 声源与感应器官之间就切 断了联系, 声波振动就无法感应器官组织, 所以, 中间介质的 地位和作用是不容忽视的 ${ }^{[2]}$ 。

\section{4 感应器官}

感应器官是接收声波能量并将其能量转换成声音信息的 器官组织。它完成了声波密码的解读和翻译工作, 让声波能量 的信息能够适应生物体的听觉器官, 从而完成了声音从声源 的发声到中间介质的传递再到感应器官的接收的全部过程。 所以, 它也是声波感应现象的最终结果。

有些动物的感应器官是耳.朵。耳朵分为外耳和内耳. 2 大 部分, 外耳:负责接收声波能量并产生耳膜振动, 振动频率被内 耳分解成各条信息, 内耳通过神经传输, 把声波的各条信息传 递给大脑神经, 经过大脑的声动细胞进行逐个解读, 然后翻译 成有效的声音。同一种声波,对于不同的生物所翻译的声音效 果是不同的, 就好比同一个单词的中英文读音不同一样, 这就 是所谓的“人有人言, 兽有兽语”的说法。

有些动物的感应器官是触须。它们的感应器官很特别, 只 用细小的须尖接收声波能量, 而用须颈去振动神经, 用须根翻 译声波密码。所以, 它们感应声波能量的能力就很弱小, 须根 也就很难翻译出有效的声音来, 从而造成它们听觉的不够发 达, 日常生活中只能依靠嗅觉来感应次声波。

任何生物(包括植物)对于声波的解读都是有先后顺序的。 对于几种同时发射的声波, 具有优先解读能量强的或较熟悉频 率的功能, 至于那些能量较弱的陌生的声波频率, 则把它们放 在稍后的位置解读或直接排余, 不去解读。听觉器官异常发达、 听觉神经又非常敏感的生物, 能够听见超声波的音域范围, 能 够迅速解读声波来源，对声波能量的冲击也能做出准确的反 应; 而听觉器官不够发达、听觉神经又反应迟钝的生物, 只能听 取次声波的音域范围，甚至不能感应到任何能量的声波冲击。

\section{5 结语}

声音形成的这 3 个环节,也决定了声波形成的前后顺序， 次声波先于声波产生, 音域范围广且能量弱, 超声波晚于声波 产生, 范围小而能量强。人们常说, 任何声源发声, 都会有次声 波和超声波形成，只不过有些声波被中间介质的能量抵消或 吸收了, 到达人耳的就只有声波。

由于声波能量的不间断冲击，声音信息就以能量形式被 感应接收,由于声波每时每刻都在发生, 所以宇宙中的声音种 类既复杂又多样。

\section{参考文献}

[1]汪勇.声波转介质跨神经传导[J].实用临床护理学,2017,2(52): 187-189.

[2] 黄杰, 杨国权,李振春, 等.TTI 介质拟声波方程数值模拟[J].物 探与化探,2018,42(1):134-143. 